\title{
On Asymptotically Lacunary Statistical Equivalent Sequences of Order $\alpha$ in Probability
}

\author{
Mahmut Işı $\mathrm{K}^{1, \star}$ and Kübra Elif Akbaş ${ }^{2,3, \star \star}$ \\ ${ }^{1}$ Faculty of Education, Harran University, Osmanbey Campus, 63190 Şanlıurfa, Turkey \\ ${ }^{2}$ Faculty of Medicine, Fırat University, 23119 Elazığ, Turkey \\ ${ }^{3}$ Faculty of Medicine, Inönü University, 44280, Malatya, Turkey
}

\begin{abstract}
In this study, we introduce and examine the concepts of asymptotically lacunary statistical equivalent of order $\alpha$ in probability and strong asymptotically lacunary equivalent of order $\alpha$ in probability. We give some relations connected to these concepts.
\end{abstract}

\section{Introduction and Background}

The idea of statistical convergence was given by Zygmund [22] in the first edition of his monograph published in Warsaw in 1935. The concept of statistical convergence was introduced by Steinhaus [21] and Fast [9] and later reintroduced by Schoenberg [19] independently. Over the years and under different names statistical convergence has been discussed in the theory of Fourier analysis, Ergodic theory, Number theory, Measure theory, Trigonometric series, Turnpike theory and Banach spaces. Later on it was further investigated from the sequence spaces point of view and linked with summability theory by Altınok et al. [1], Connor [4], Çolak [5], Das et al. [6], Et et al. ([7],[20]) Fridy [11], Işık et al. ([13],[14],[15]) and many others. In recent years, generalizations of statistical convergence have appeared in the study of strong integral summability and the structure of ideals of bounded continuous functions on locally compact spaces. Statistical convergence and its generalizations are also connected with subsets of the Stone-Čech compactification of the natural numbers. Moreover, statistical convergence is closely related to the concept of convergence in probability.

Marouf [16] introduced definitions for asymptotically equivalent sequences and asymptotic regular matrices. Patterson [17] extend these concepts by presenting an asymptotically statistically equivalent analog of these definitions and natural regularity conditions for nonnegative summability matrices. Later on asymptotically equivalent sequences have been studied in ([2], [3],[18]).

By a lacunary sequence we mean an increasing integer sequence $\theta=\left(k_{r}\right)$ such that $h_{r}=$ $\left(k_{r}-k_{r-1}\right) \rightarrow \infty$ as $r \rightarrow \infty$. Throught this paper the intervals determined by $\theta$ will be denoted by $I_{r}=\left(k_{r-1}, k_{r}\right]$ and the ratio $\frac{k_{r}}{k_{r-1}}$ will be abbreviated by $q_{r}$. Recently lacunary sequences have been studied in ([8],[10],[12],[20]).

Let $X_{n}(n \in \mathbb{N})$ be a random variable which is defined on a given event space $S$ with respect to a given class of events $\Delta$ and a probability function $P: \Delta \rightarrow \mathbb{R}$, then we say that $X_{1}, X_{2}, X_{3}, \ldots, X_{n} \ldots$ is a sequence of random variables. A sequence of random variables is denoted by $\left\{X_{n}\right\}_{n \in \mathbb{N}}$.

\footnotetext{
$\star$ e-mail: misik63@yahoo.com

$\star \star$ e-mail: elifet41@gmail.com
} 
Definition 1 Let $(S, \Delta, P)$ be a probability space, $\theta=\left(k_{r}\right)$ be a lacunary sequence, $\alpha \in(0,1]$ be any real number. Two nonnegative sequences of random variables $\left\{X_{n}\right\}_{n \in \mathbb{N}}$ and $\left\{Y_{n}\right\}_{n \in \mathbb{N}}$ are said to be asymptotically lacunary statistical equivalent of order $\alpha$ in probability provided that for every $\varepsilon, \delta>0$,

$$
\lim _{r} \frac{1}{h_{r}^{\alpha}}\left|\left\{k \in I_{r}: P\left(\left|\frac{X_{k}}{Y_{k}}-X\right| \geq \varepsilon\right) \geq \delta\right\}\right|=0 .
$$

In this case we write $X \stackrel{P S_{\theta}^{\alpha}}{\sim} Y$.

Definition 2 Let $(S, \Delta, P)$ be a probability space, $\theta=\left(k_{r}\right)$ be a lacunary sequence and let $\alpha$ be any real number such that $\alpha>0$. Two nonnegative sequences of random variables $\left\{X_{n}\right\}_{n \in \mathbb{N}}$ and $\left\{Y_{n}\right\}_{n \in \mathbb{N}}$ are said to be strong asymptotically lacunary equivalent of order $\alpha$ in probability provided that for every $\varepsilon>0$,

$$
\lim _{r \rightarrow \infty} \frac{1}{h_{r}^{\alpha}} \sum_{k \in I_{r}} P\left(\left|\frac{X_{k}}{Y_{k}}-X\right| \geq \varepsilon\right)=0 .
$$

In this case we write $X \stackrel{P N_{\theta}^{\alpha}}{\sim} Y$.

\section{Main Results}

We give the following results without proof.

Theorem 1 Let $\theta=\left(k_{r}\right)$ be a lacunary sequence and let $\alpha$ and $\beta$ be fixed real numbers such that $0<\alpha \leq \beta \leq 1$, then $X \stackrel{P N_{\theta}^{\alpha}}{\sim} Y$ implies $X \stackrel{P S_{\theta}^{\beta}}{\sim} Y$.

Theorem 2 Let $\theta=\left(k_{r}\right)$ be a lacunary sequence and let $\alpha$ and $\beta$ be fixed real numbers such that $0<\alpha \leq \beta \leq 1$. If $\liminf _{r} q_{r}>1$, then $X \stackrel{P S^{\alpha}}{\sim} Y$ implies $X \stackrel{P S_{\theta}^{\beta}}{\sim} Y$.

Theorem 3 Let $\theta=\left(k_{r}\right)$ be a lacunary sequence and $\alpha \in(0,1]$. If $\lim \sup _{r} q_{r}<\infty$, then $X \stackrel{P S_{\theta}^{\alpha}}{\sim} Y$ implies $X \stackrel{P S}{\sim} Y$.

Theorem 4 Let $\theta=\left(k_{r}\right)$ be a lacunary sequence and let $\alpha$ be fixed real number such that $0<\alpha \leq 1$. If $\liminf { }_{r} q_{r}>1$, then $X \stackrel{P N^{\alpha}}{\sim} Y$ implies $X \stackrel{P N_{\theta}^{\alpha}}{\sim} Y$.

Theorem 5 Let $\theta=\left(k_{r}\right)$ be a lacunary sequence. If $\lim \sup _{r} \frac{k_{r}}{k_{r-1}^{\alpha}}<\infty$, then $X \stackrel{P N_{\theta}}{\sim} Y$ implies $X \stackrel{P N^{\alpha}}{\sim} Y$.

Theorem 6 Let $\theta=\left(k_{r}\right)$ be a lacunary sequence and $\alpha \in(0,1]$. If

$$
\lim _{r \rightarrow \infty} \inf \frac{h_{r}^{\alpha}}{k_{r}}>0
$$

then $X \stackrel{P S}{\sim} Y$ implies $X \stackrel{P S_{\theta}^{\alpha}}{\sim} Y$.

Now let $\theta=\left(k_{r}\right)$ and $\theta^{\prime}=\left(s_{r}\right)$ be two lacunary sequences such that $I_{r} \subseteq J_{r}$ for all $r \in \mathbb{N}, \alpha$ and $\beta$ be fixed real numbers such that $0<\alpha \leq \beta \leq 1$. Now we give some inclusion relations between the concepts of asymptotically lacunary statistical equivalent of order $\alpha$ in probability and strong asymptotically lacunary equivalent of order $\beta$ in probability. 
Theorem 7 Let $\theta=\left(k_{r}\right)$ and $\theta^{\prime}=\left(s_{r}\right)$ be two lacunary sequences such that $I_{r} \subset J_{r}$ for all $r \in \mathbb{N}$ and let $\alpha$ and $\beta$ be fixed real numbers such that $0<\alpha \leq \beta \leq 1$. Then each of the following assertions holds true

(i) If

$$
\lim _{r \rightarrow \infty} \inf \frac{h_{r}^{\alpha}}{\ell_{r}^{\beta}}>0
$$

then $X \stackrel{P S_{\theta^{\prime}}^{\beta}}{\sim} Y$ implies $X \stackrel{P S_{\theta}^{\alpha}}{\sim} Y$.

(ii) If

$$
\lim _{r \rightarrow \infty} \frac{\ell_{r}}{h_{r}^{\beta}}=1
$$

then $X \stackrel{P S_{\theta}^{\alpha}}{\sim} Y$ implies $X \stackrel{P S_{\theta^{\prime}}^{\beta}}{\sim} Y$.

where $I_{r}=\left(k_{r-1}, k_{r}\right], J_{r}=\left(s_{r-1}, s_{r}\right], h_{r}=k_{r}-k_{r-1}$ and $\ell_{r}=s_{r}-s_{r-1}$.

Proof (i) Suppose that $I_{r} \subset J_{r}$ for all $r \in \mathbb{N}$ and let (1) be satisfied. For given $\varepsilon, \delta>0$ we have

$$
\left\{k \in J_{r}: P\left(\left|\frac{X_{k}}{Y_{k}}-X\right| \geq \varepsilon\right) \geq \delta\right\} \supseteq\left\{k \in I_{r}: P\left(\left|\frac{X_{k}}{Y_{k}}-X\right| \geq \varepsilon\right) \geq \delta\right\}
$$

and so

$$
\frac{1}{\ell_{r}^{\beta}}\left|\left\{k \in J_{r}: P\left(\left|\frac{X_{k}}{Y_{k}}-X\right| \geq \varepsilon\right) \geq \delta\right\}\right| \geq \frac{h_{r}^{\alpha}}{\ell_{r}^{\beta}} \frac{1}{h_{r}^{\alpha}}\left|\left\{k \in I_{r}: P\left(\left|\frac{X_{k}}{Y_{k}}-X\right| \geq \varepsilon\right) \geq \delta\right\}\right|
$$

for all $r \in \mathbb{N}$. Now taking the limit as $r \rightarrow \infty$ in the last inequality and using (1) we get $X \stackrel{P S_{\theta}^{\alpha}}{\sim} Y$.

(ii) Let $X \stackrel{P S_{\theta}^{\alpha}}{\sim} Y$ and (2) be satisfied. Since $I_{r} \subset J_{r}$, for $\varepsilon, \delta>0$ we may write

$$
\begin{aligned}
\frac{1}{\ell_{r}^{\beta}}\left|\left\{k \in J_{r}: P\left(\left|\frac{X_{k}}{Y_{k}}-X\right| \geq \varepsilon\right) \geq \delta\right\}\right|= & \frac{1}{\ell_{r}^{\beta}}\left|\left\{s_{r-1}<k \leq k_{r-1}: P\left(\left|\frac{X_{k}}{Y_{k}}-X\right| \geq \varepsilon\right) \geq \delta\right\}\right| \\
& +\frac{1}{\ell_{r}^{\beta}}\left|\left\{k_{r}<k \leq s_{r}: P\left(\left|\frac{X_{k}}{Y_{k}}-X\right| \geq \varepsilon\right) \geq \delta\right\}\right| \\
& +\frac{1}{\ell_{r}^{\beta}}\left|\left\{k_{r-1}<k \leq k_{r}: P\left(\left|\frac{X_{k}}{Y_{k}}-X\right| \geq \varepsilon\right) \geq \delta\right\}\right| \\
\leq & \frac{k_{r-1}-s_{r-1}}{\ell_{r}^{\beta}}+\frac{s_{r}-k_{r}}{\ell_{r}^{\beta}} \\
& +\frac{1}{\ell_{r}^{\beta}}\left|\left\{k \in I_{r}: P\left(\left|\frac{X_{k}}{Y_{k}}-X\right| \geq \varepsilon\right) \geq \delta\right\}\right| \\
\leq & \left(\frac{\ell_{r}}{h_{r}^{\beta}}-1\right)+\frac{1}{h_{r}^{\alpha}}\left|\left\{k \in I_{r}: P\left(\left|\frac{X_{k}}{Y_{k}}-X\right| \geq \varepsilon\right) \geq \delta\right\}\right|
\end{aligned}
$$

for all $r \in \mathbb{N}$. Hence $X \stackrel{P S_{\beta^{\prime}}^{\beta}}{\sim} Y$.

Theorem 8 Let $\theta=\left(k_{r}\right)$ and $\theta^{\prime}=\left(s_{r}\right)$ be two lacunary sequences such that $I_{r} \subseteq J_{r}$ for all $r \in \mathbb{N}, \alpha$ and $\beta$ be fixed real numbers such that $0<\alpha \leq \beta \leq 1$. Then we have

(i) Let the condition (1) holds, then $X \stackrel{P N_{\theta^{\prime}}^{\beta}}{\sim} Y$ implies $X \stackrel{P N_{\theta}^{\alpha}}{\sim} Y$, 
(ii) Let the condition (2) holds, $\left\{X_{n}\right\}_{n \in \mathbb{N}}$ and $\left\{Y_{n}\right\}_{n \in \mathbb{N}}$ be two bounded sequences of random variables, then $X \stackrel{P N_{\theta}^{\alpha}}{\sim} Y$ implies $X \stackrel{P N_{\theta^{\prime}}^{\beta}}{\sim} Y$.

Proof Omitted.

Theorem 9 Let $\theta=\left(k_{r}\right)$ and $\theta^{\prime}=\left(s_{r}\right)$ be two lacunary sequences such that $I_{r} \subseteq J_{r}$ for all $r \in \mathbb{N}, \alpha$ and $\beta$ be fixed real numbers such that $0<\alpha \leq \beta \leq 1$.

(i) Let the condition (1) holds, then $X \stackrel{P N_{\theta^{\prime}}^{\beta}}{\sim} Y$ implies $X \stackrel{P S_{\theta}^{\alpha}}{\sim} Y$.

(ii) Let the condition (2) holds and let $\left\{X_{n}\right\}_{n \in \mathbb{N}}$ and $\left\{Y_{n}\right\}_{n \in \mathbb{N}}$ be two bounded sequences of random variables then $X \stackrel{P S_{\theta}^{\alpha}}{\sim} Y$ implies $X \stackrel{P N_{\theta^{\prime}}^{\beta}}{\sim} Y$.

Proof $(i)$ For any sequence $\left\{X_{n}\right\}_{n \in \mathbb{N}}$ of random variables and $\varepsilon, \delta>0$, we have

$$
\begin{aligned}
\sum_{k \in J_{r}} P\left(\left|\frac{X_{k}}{Y_{k}}-X\right| \geq \varepsilon\right) & \geq \sum_{k \in I_{r}} P\left(\left|\frac{X_{k}}{Y_{k}}-X\right| \geq \varepsilon\right) \\
& P\left(\left|\frac{X_{k}}{Y_{k}}-X\right| \geq \varepsilon\right) \geq \delta \\
\geq & \left|\left\{k \in I_{r}: P\left(\left|\frac{X_{k}}{Y_{k}}-X\right| \geq \varepsilon\right) \geq \delta\right\}\right| \varepsilon
\end{aligned}
$$

and so that

$$
\frac{1}{\ell_{r}^{\beta}} \sum_{k \in J_{r}} P\left(\left|\frac{X_{k}}{Y_{k}}-X\right| \geq \varepsilon\right) \geq \frac{h_{r}^{\alpha}}{\ell_{r}^{\beta}} \frac{1}{h_{r}^{\alpha}}\left|\left\{k \in I_{r}: P\left(\left|\frac{X_{k}}{Y_{k}}-X\right| \geq \varepsilon\right) \geq \delta\right\}\right| \varepsilon .
$$

Hence $X \stackrel{P S_{\theta}^{\alpha}}{\sim} Y$.

ii) Omitted.

\section{References}

[1] H. Altınok, Y. Altın, M. Işık, Iran. J. Fuzzy Syst., 9(2), 63-73 (2012)

[2] M. Başarır, S. Altundağ, Filomat, 22(1), 161-172 (2008)

[3] N. L. Braha, Appl. Math. Comput., 219(1), 280-288 (2012)

[4] J. S. Connor, Analysis, 8, 47-63 (1988)

[5] R. Çolak, Modern Methods in Analysis and Its Applications, New Delhi, India: Anamaya Pub, 121-129 (2010)

[6] P. Das, S. Ghosal, S. Som, Arab J. Math. Sci., 21(2), 253-265 (2015)

[7] M. Et, H. Altınok, Y. Altın, Appl. Math. Comput., 154(1), 167-173 (2004)

[8] M. Et, H. Şengül, Filomat, 28(8), 1593-1602 (2014)

[9] H. Fast, Colloq. Math., 2, 241-244 (1951)

[10] A. R. Freedman, J. J. Sember, M. Raphael, Proc. Lond. Math. Soc., 37, 508-520 (1978)

[11] J. A. Fridy, Analysis, 5 , 301-313 (1985)

[12] J. A. Fridy, C. Orhan, Pacific J. Math. 160, 43-51 (1993)

[13] M. Işık, Math. Slovaca, 61(5), 779-788 (2011)

[14] M. Işık, J. Inequal. Appl., 2010, Art. ID 457892, 7 pp. (2010) 
[15] M. Iş1k, K. E. Et, AIP Conference Proceedings, 1676(1), 020045 (2015)

[16] M. S. Marouf, Internat. J. Math. Math. Sci., 16(4), 755-762 (1993)

[17] R. F. Patterson, Demonstratio Math., 36(1), 149-153 (2003)

[18] R. Savaş, M. Başarır, Filomat, 20(1), 35-42 (2006)

[19] I. J. Schoenberg, Amer. Math. Monthly, 66, 361-375 (1959)

[20] H. Şengül, M. Et, Acta Math. Sci. Ser. B Engl. Ed., 34(2), 473-482 (2014)

[21] H. Steinhaus, Colloquium Mathematicum, 2, 73-74 (1951)

[22] A. Zygmund, Trigonometric Series, (Cambridge University Press, Cambridge, UK, 1979) 